\title{
Ontology reuse for multiagent systems development through pattern classification
}

\begin{abstract}
Ontologies play a crucial role in multiagent systems (MASs) development, especially for domain knowledge modeling, interaction specifications, and behavioral aspect representation. Domain-specific ontologies can be developed in an ad hoc or systematic manner through the incorporation of ontology development steps on the basis of agent-oriented methodologies. Developing such ontologies, however, is challenging because of the extensive amounts of knowledge and experience required. Moreover, since many ontologies cater for very specific domains, the question arises of whether some can be reused for faster systems development. This paper attempts to answer this question by proposing an ontology pattern classification scheme to allow the reuse of existing ontology knowledge for MAS development. Specifically, ontology patterns relevant to the design problem at hand are identified through the pattern classification scheme. These patterns are then reused and shared among agent software communities during the system development phase. The effectiveness of the proposed approach is validated using a restaurant-finder MAS case study. Our findings suggest that utilization of the classified ontology patterns reduces development time and complexity when dealing with domain-specific applications. The scheme also seems useful for software practitioners, where searching and reusing the patterns can easily be done during the analysis, design, and implementation of MAS development.
\end{abstract}

Keyword : Multi-agent system; Ontology; Ontology pattern; Ontology reuse 